\title{
Review
}

Ophthalmic

Research

\section{Cataract Surgery and Intraocular Pressure}

\author{
Diana Melancia ${ }^{c}$ Luis Abegão Pinto ${ }^{a-c}$ Carlos Marques-Neves ${ }^{b, c}$ \\ Departments of a Pharmacology and Neurosciences and ${ }^{b}$ Ophthalmology, ${ }^{c}$ Faculty of Medicine, Lisbon University, \\ Lisbon, Portugal
}

\section{Key Words \\ Cataract surgery · Glaucoma · Intraocular pressure · \\ Phacoemulsification · Trabeculectomy · Combined surgery}

\begin{abstract}
Cataract surgery is one of the most performed surgeries in the developed world. In addition to its significant impact on visual acuity, phacoemulsification has been hailed as a potential intraocular pressure (IOP)-lowering procedure. While current evidence suggests an overall significant and sustained decrease in IOP to exist after cataract surgery, the specific ocular characteristics that could help predict which patients are likely to benefit from this IOP-lowering effect remain unclear. This definition is important in glaucoma patients if this surgery is to be used in the treatment for this disease. Our review aims to summarize the literature on the subject, depicting possible mechanisms behind this IOP decrease, which type of patients are more likely to benefit from this surgery for IOP-lowering purposes and ultimately help optimizing disease management for the increasing number of patients with concomitant glaucoma and cataract.
\end{abstract}

(c) 2015 S. Karger AG, Basel

\section{Introduction}

Cataract and glaucoma are ranked as the leading causes of blindness worldwide (51 and $8 \%$, respectively) [1]. In developed countries, glaucoma is the second leading cause of irreversible blindness (after diabetic retinopathy) and this burden tends to increase as the population ages [2]. A similar trend is seen with cataract, whose prevalence is also age-related - the global prevalence of $15.5 \%$ rises to $45.9 \%$ in those over 75 years and is expected to duplicate by 2020 [3]. Unsurprisingly, both diseases frequently coexist in the elderly population in a proportion that is likely to increase.

Given this data, cataract surgery rises as one of the most common surgical procedures performed worldwide and it has been suggested to be of clinical benefit for both diseases. Besides removing the opacified lens, cataract surgery has been suggested to reduce intraocular pressure (IOP) in eyes either with or without glaucoma, although with variable magnitude and influenced by several factors, including anterior chamber anatomy and angle configuration (open-angle vs. angle-closure) [4]. Also, it has the ability to increase the accuracy of functional and structural analyses currently used for diagnosing and evaluating glaucoma and its progression, since a visually significant cataract may act as a confounder. Therefore, it seems reasonable to assume that a conjoint treatment could be established, with cataract surgery being part of glaucoma treating algorithms. However, questions remain on how it would best fit in the clinical management of these patients.

This review focuses on how cataract surgery might influence IOP control in both healthy individuals and glaucoma patients with cataract. In this particular setting, intrinsic glaucoma anatomical and physiological factors

\section{KARGER 125}

2015 S. Karger AG, Base

0030-3747/15/0533-0141\$39.50/0
Carlos Marques-Neves, $\mathrm{MD}, \mathrm{PhD}$

Department of Ophthalmology

Faculty of Medicine, Lisbon University

Av. Prof. Egas Moniz, PT-1649-035 Lisbon (Portugal)

E-Mail bombordo.seven@gmail.com 
Table 1. Clinical studies reflecting the effect of lens extraction on IOP in patients without glaucoma, ocular hypertension, OAG, CACG and acute ACG

\begin{tabular}{|c|c|c|c|c|c|}
\hline Authors & $\mathrm{n}$ & Follow-up & $\begin{array}{l}\text { IOP pre } \rightarrow \text { post, } \\
\mathrm{mm} \mathrm{Hg}\end{array}$ & $\Delta \mathrm{IOP}$ & $\mathrm{p}$ value \\
\hline \multicolumn{6}{|l|}{ Without glaucoma } \\
\hline Pohjalainen [6], 2001 & 137 & 25 months & $16.3 \rightarrow 12.7$ & -3.4 & 0.001 \\
\hline Shingleton [7], 2006 & 59 & 60 months & $15.9 \rightarrow 13.4$ & -1.5 & $<0.0001$ \\
\hline \multicolumn{6}{|l|}{ Ocular hypertension } \\
\hline Mansberger [5], 2012 & 63 & 38 months & $23.9 \rightarrow 19.8$ & -4.1 & $<0.001$ \\
\hline \multicolumn{6}{|l|}{ POAG } \\
\hline Kim [14], 1999 & 31 & 16 months & $18.1 \rightarrow 15.2$ & -2.9 & $<0.001$ \\
\hline Shingleton [15], 1999 & 61 & 12 months & $17.0 \rightarrow 15.9$ & -1.1 & n.s. \\
\hline Pohjalainen [16], 2001 & 38 & 26 months & $18.4 \rightarrow 15.1$ & -3.3 & 0.001 \\
\hline Hayashi [10], 2001 & 68 & 24 months & $20.7 \rightarrow 15.2$ & -5.3 & $<0.0001$ \\
\hline Lai [11], 2004 & 34 & 20 months & $20.4 \rightarrow 15.7$ & -4.7 & $<0.05$ \\
\hline Mathalone [17], 2005 & 24 & 24 months & $17.0 \rightarrow 15.1$ & -1.9 & n.s. \\
\hline Shingleton [7], 2006 & 55 & 60 months & $18.4 \rightarrow 16.6$ & -1.8 & 0.005 \\
\hline Poley [18], 2009 & 124 & 10 years & $17.8 \rightarrow 15.1$ & -2.7 & $<0.001$ \\
\hline \multicolumn{6}{|l|}{ CACG } \\
\hline Hayashi [10], 2001 & 68 & 24 months & $21.0 \rightarrow 15.0$ & -6.0 & $<0.001$ \\
\hline Lai [11], 2004 & 31 & 20 months & $27.4 \rightarrow 14.8$ & -12.7 & $<0.05$ \\
\hline Liu [12], 2006 & 29 & 3 months & $\mathrm{ND}(>20.0) \rightarrow 14.3$ & ND & ND \\
\hline Lai [13], 2006 & 21 & 21 months & $19.7 \rightarrow 15.5$ & -4.2 & 0.02 \\
\hline \multicolumn{6}{|l|}{ Acute ACG } \\
\hline Jacobi [8], 2002 & 43 & 24 months & $40.5 \rightarrow 17.80$ & -22.70 & ND \\
\hline $\mathrm{Su}[9], 2011$ & 16 & 3 months & $48.81 \rightarrow 10.70$ & -38.09 & $<0.001$ \\
\hline
\end{tabular}

that influence IOP and that may be potentially predictive of the effect that lensectomy might have on IOP control will be focused on. For open-angle glaucoma (OAG) and angle-closure glaucoma (ACG), current surgical options will be compared to each other, discussing the clinical aspects that influence the choice of surgical treatment.

\section{Ocular Biometric Parameters}

\section{Predicting Post-Operative IOP}

In the 1970s, Bigger and Becker reported a lower IOP after cataract extraction in glaucoma patients [5]. Since then, and particularly in the last decade, many studies have consistently shown a variable IOP decrease after cataract surgery (table 1) [5-18].

Pre-operative angle configuration is pointed out as one of the main factors contributing to this variability, as higher IOP reductions are observed with partially or completely closed angles. Besides angle anatomy, many other factors were independently related to IOP reduc- tion of cataract surgery including aqueous humour dynamics, ocular comorbidities and, most importantly, preoperative IOP.

After lens removal, even eyes without glaucoma experience anatomical changes in the anterior chamber, and many biometrical factors are modified. For instance, an increase in anterior chamber depth (ACD), angle opening distance and anterior chamber area are to be expected [19-21]. These anatomical factors are particularly relevant in ACG, typically characterized by a narrow angle and a shallow anterior chamber that progressively decrease as the lens becomes thicker and more anteriorly positioned. When submitted to phacoemulsification, ACG patients undergo a significant increase in ACD and angle width when comparing with OAG patients or even with control patients [21]. These biometrical changes correlate with IOP in all groups, with a more pronounced IOP reduction $(\mathrm{p}=0.02)$ in ACG patients than in OAG patients or the control group [10].

Given these anatomical and biometric parameters, some predictive indexes of post-operative IOP were cre- 
Table 2. Rate of conversion of ocular hypertension to glaucoma or normal IOP [21]

\begin{tabular}{|c|c|c|c|c|c|c|c|c|}
\hline Study & $\mathrm{n}$ & $\begin{array}{l}\text { Follow-up } \\
\text { years }\end{array}$ & Treatment & $\begin{array}{l}\Delta \mathrm{IOP} \\
\mathrm{mm} \mathrm{Hg}\end{array}$ & \multicolumn{2}{|l|}{ Conversion to glaucoma } & \multicolumn{2}{|c|}{ Conversion to normal IOP } \\
\hline OHTS [5] & 817 & 5.0 & medication & $21-32$ & $\downarrow$ visual field & 4.4 & IOP $<21$ & 0.0 \\
\hline Poley $[18,27]$ & 81 & 4.1 & Phaco + IOL & $20-31$ & start of medical treatment & 1.1 & IOP $<20$ & 74.7 \\
\hline
\end{tabular}

OHTS = Ocular Hypertensive Treatment Study; Phaco = phacoemulsification; IOL = intraocular lens. Adapted from Poley et al. [18].

ated. In 2005, Issa et al. [22] developed a predictive index for IOP reduction in patients without glaucoma based on two anatomical factors - IOP and ACD - and concluded that it correlated better with IOP variation than each of the parameters individually considered. Also, Liu et al. [23] suggested another formula for eyes with ACG based on IOP and ACD that would allow to predict the IOP variation after cataract surgery [IOP $=6.354+0.186$ (IOP $\left.\times \mathrm{ACD}) ; \mathrm{R}^{2}=49 \%\right]$. One year after the surgery, the product of IOP and ACD predicted that eyes with a ratio of $<35$ have a better probability of achieving an IOP $\leq 12 \mathrm{~mm}$ $\mathrm{Hg}$ post-operatively. Although these indexes are neither validated nor globally implemented, they might be helpful when making decisions regarding which surgical procedure to perform, whether a simple lens extraction would suffice to control the IOP or a more invasive (yet more effective) procedure would be necessary. Finally, many other biometric factors have been proposed as independent predictors of post-operative IOP - anterior chamber area, anterior chamber width, lens thickness but their usefulness warrants further investigation [20, $24,25]$.

\section{IOP Reduction in Anterior Chambers with an Open Angle}

IOP decrease may not only depend on anatomical factors relating to narrow angles. Poley et al. [18] suggest that lens removal allows the posterior capsule to move posteriorly, dislodging the zonula over the ciliary body with a consequent widening of Schlemm's canal and aqueous humour drainage improvement. Another proposed mechanism states that the ultrasounds used in the phacoemulsification procedure are responsible for an abrupt rise in the anterior chamber pressure, producing inflammatory cytokines (mostly IL-1) that stimulate metalloproteinase production and trabecular meshwork remodelling, facilitating humour drainage [26]. As such, and also as stated by Poley et al. [27], pre-operative IOP is the best predictor of post-operative IOP, as the IOP variation in OAG patients appears to be proportional to the magnitude of the pre-operative IOP [28].

\section{Ocular Hypertension}

Ocular hypertension is defined as an IOP higher than normal $(21 \mathrm{~mm} \mathrm{Hg})$ in the absence of visual field loss or optic nerve damage. It is, by itself, the main risk factor for progression to glaucoma. According to the Ocular Hypertensive Treatment Study [5], phacoemulsification with intraocular lens implantation decreases IOP in this subset of patients proportionally to their pre-operative IOP. Poley et al. $[18,27]$ evaluated the impact of phacoemulsification on the progression of ocular hypertension for 10 years and concluded that cataract surgery, by providing a bigger IOP reduction, might be responsible for a lower progression to glaucoma (table 2). However, evidence is still missing on the role cataract surgery might have as a preventive measure of ocular hypertension progression to glaucoma.

\section{Angle-Closure Glaucoma}

Primary angle closure is defined as the presence of iridotrabecular contact impairing aqueous humour outflow through a diffuse obstruction of the trabecular meshwork and it usually results from a shallow anterior chamber and angle-crowding of the anterior segment. The occurrence of a closed angle is more likely as the distance between iris and trabecular meshwork decreases, and the risk of iridotrabecular contact rises exponentially from an angle opening of $20^{\circ}$ [29]. Owing to the intrinsic role of the lens in the anterior chamber anatomy in patients with primary angle closure, its surgical extraction might be useful in the treatment and management of acute and chronic ACG. 
Table 3. Comparison of LPI and phacoemulsification with intraocular lens implantation

\begin{tabular}{lcccc}
\hline & LPI & Phaco & p value & Study \\
\hline Long-term IOP control & & & & \\
$\quad>21 \mathrm{~mm} \mathrm{Hg}$ & $46.7 \%$ & $3.3 \%$ & $<0.0001$ & Lam [30], 2008 \\
$\quad<22 \mathrm{~mm} \mathrm{Hg}$ & $61.1 \%$ & $89.5 \%$ & 0.034 & Husain [31], 2012 \\
Angle-opening (Shaffer) & 0.73 & 2.10 & $<0.0001$ & Lam [30], 2008 \\
Medication necessary to control IOP & 0.90 & 0.03 & $<0.0001$ & Lam [30], 2008 \\
IOP at 18 months, mm Hg & 15.0 & 12.6 & 0.009 & Lam [30], 2008 \\
PAS, degrees & 228.6 & 101.3 & $<0.0001$ & Lam [30], 2008 \\
\hline
\end{tabular}

Phaco $=$ Phacoemulsification with intraocular lens implantation.

With an unresolved acute angle closure crisis, lens extraction results in a steep reduction in IOP (table 1). As a thick lens boosts pupillary block and consequent angle closure, its removal allows for expansion of anterior chamber dimensions and angle width, promoting aqueous humour outflow and IOP reduction. Also, even when compared with laser peripheral iridotomy (LPI), this treatment modality presents with further advantages (table 3): better long-term IOP control after resolution of an acute crisis, lesser need for long-term medication in order to maintain an optimal IOP, wider angle and fewer peripheral anterior synechiae (PAS) formation, with a smaller risk of a second angle closure crisis or progression to chronic angle closure, which is approximately $58.1 \%$ after LPI [30, 31]. It is, however, a riskier procedure due to the shallow anterior chamber, the vulnerability of the corneal endothelia and an atonic iris [32].

In the absence of a cataract or in eyes with good visual acuity, the clinical benefit of cataract surgery is still under debate and LPI appears to have the best risk-to-benefit ratio. Nonetheless, when a cataract is present, lens extraction is suggested as the best choice once the acute crisis is duly aborted [32]. Also, when IOP at presentation is higher than $55 \mathrm{~mm} \mathrm{Hg}$ (which is an independent factor of long-term uncontrolled IOP), patients are less likely to require IOP-lowering therapy if treated with early cataract extraction, when comparing with LPI [30].

In the presence of PAS, the anterior chamber angle becomes chronically closed with permanent obstruction of aqueous humour drainage. IOP is now almost entirely dependent on the extent of trabecular meshwork damage, which can be directly inflicted by the PAS themselves or derive indirectly from the elevated IOP - whether hypertensive spikes or continuously high pressure. IOP becomes continuously elevated when more than $180^{\circ}$ of angle are occluded by PAS and when it surpasses $270^{\circ}$, med- ical therapy is usually ineffective and filtering surgery is required [33].

Traditionally, the approach to the patient with primary ACG is a stepped combination of medical and laser therapy; glaucoma surgery is indicated as a second-line option when these treatments fail. However, cataract extraction is being increasingly considered for primary ACG management owing to its capacity to restore the anatomy of the anterior chamber with the lens extraction, often without the need for further medication.

In primary ACG patients with a clear lens, both phacoemulsification and trabeculectomy are effective in reducing IOP (reduction of $8.4 \mathrm{~mm} \mathrm{Hg}$ or $34 \%$ for phacoemulsification vs. $8.9 \mathrm{~mm} \mathrm{Hg}$ or $36 \%$ for trabeculectomy; $\mathrm{p}>$ $0.05)$. Trabeculectomy-treated patients seemed to have a smaller need for additional drugs than phacoemulsification-treated patients (although not statically significant, $\mathrm{p}=0.16$ ); likewise, trabeculectomy was also associated with significantly more surgical complications than phacotrabeculectomy ( 46 vs. $4 \%, p=0.001$ ) [34]. The most frequent long-term complication is cataract formation (33\%), adding the need for another surgical procedure to restore the visual acuity [34]. Also, it is estimated that half the patients with chronic angle-closure glaucoma (CACG) undergoing trabeculectomy will need lens extraction within 5 years [35].

Based on these clinical trials, it has been a subject of debate whether early clear lens extraction would be of any clinical benefit in controlling moderate CACG or even in CACG prevention. In a 2006 meta-analysis, Friedman and Vedula [36] concluded that there was no solid evidence supporting this clear lens extraction for glaucoma treatment. Nowadays, however, recent clinical trials contradict this view and defend phacoemulsification as a valuable option based on it being a safer, faster and more economical procedure than every other surgical proce- 
dure for CACG. By contrast, there is currently no role for clear lens extraction for CACG prevention in patients with angle-closure suspect or chronic angle closure [37]. In an attempt to answer this question, the EAGLE trial (The Effectiveness of Early Lens Extraction with Intraocular Lens Implantation for the Treatment of Primary Angle-Closure Glaucoma) is currently under way. Beginning in 2011, it is a multicentric prospective randomized clinical trial comparing clear-lens extraction and LPI in patients with newly diagnosed chronic angle-closure or CACG [38]. The primary outcomes include IOP, patient's quality of life and cost-effectiveness, with a followup of 3 years, after which we might be able to confirm phacoemulsification as a valuable option for the current practice of CACG treatment.

Besides phacoemulsification and trabeculectomy, there is a third surgical option combining both procedures: phacotrabeculectomy or combined surgery. When compared with phacoemulsification, combined surgery seems to be slightly more effective for IOP control ( $p>$ 0.05 ) with fewer medications. Likewise, the rate of surgical complications is significantly higher in the combined surgery group, as well as the optic neuropathy progression rate $[39,40]$. Notwithstanding the need for a smaller amount of medication after trabeculectomy and the greater decrease in IOP following combined surgery, the higher rate of surgical complications outweighs this small benefit. In situations where drug reduction is a priority, trabeculectomy remains the more appropriate option [41]. Several authors defend that in the presence of an advanced glaucoma combined surgery is the best option, since it offers a better IOP control than phacoemulsification and, when compared to trabeculectomy, has comparable efficacy in controlling IOP and reducing the requirement for glaucoma drugs with a smaller re-intervention rate [42].

\section{Open-Angle Glaucoma}

IOP increase in OAG is not related to angle crowding factors but rather due to aqueous humour drainage impairment at the trabecular meshwork level. Medical therapy is the first line of treatment in the majority of cases, with filtering surgery playing a role in patients where target IOP is either not achieved or is not likely to be sustained in cases of young patients or poorly tolerated medical therapy. However, filtering surgery is not the only surgery glaucoma patients are likely to have. In fact, $30 \%$ of all the patients who underwent cataract surgery in the
US under Medicare were reported to have concomitant glaucoma [43]. This significant epidemiological factor makes it even more important to incorporate this surgery in the practice patterns of glaucoma management. Similarly to CACG, there are three surgical options to consider: phacoemulsification, trabeculectomy or combined surgery (phacotrabeculectomy). Depending on glaucoma severity, patient approach should be individually tailored:

Mild Glaucoma. For a patient with a troublesome cataract but a mild, well-controlled glaucoma requiring few medications and minor optic nerve damage, phacoemulsification alone is a reliable option. Lens removal improves visual acuity and may allow for better disease evaluation and follow-up. Furthermore, there is a possibility of a phacoemulsification-related small IOP-lowering effect, aiding in IOP control - average reduction of 1.5$3.0 \mathrm{~mm} \mathrm{Hg}$ (table 1). However, there is still a risk of complications associated with this surgery, notably hypertensive spikes.

Moderate Glaucoma. In this group of patients, clinical decisions are particularly dependent on individual characteristics. Phacoemulsification remains a valuable surgical option in patients with an otherwise controlled IOP, although requiring a higher degree of attention because of the more frequent hypertensive spikes. However, in a patient whose IOP is above target, the pros and cons of a combined surgery should be discussed with the patient. On the one hand, combining cataract surgery and trabeculectomy would allow to correct two problems with a single operative procedure (since cataract surgery after filtering surgery is almost inevitable) and would avoid the hypertensive spikes after phacoemulsification. On the other hand, combined surgery is associated with more post-operative complications and a not so significant IOP decrease (if compared with trabeculectomy alone) [44, 45]. It should be noted, however, that patients with moderate glaucoma tend to have a higher IOP target, which would make them good candidates for a one-time combined procedure. In the presence of an incipient cataract and moderate glaucoma or when IOP is not within the ideal range, glaucoma control is the priority. First-line surgical approach is trabeculectomy, but other less cataractogenic procedures might be considered, particularly non-penetrating procedures such as deep sclerectomy and viscocanalostomy, despite its smaller success rate when compared to trabeculectomy [46].

Advanced Glaucoma. When there is severe glaucoma, there is a high risk of visual acuity loss due to IOP spikes or IOP fluctuations and the priority is a more aggressive IOP-lowering strategy. The best surgical option is trab- 
eculectomy with posterior cataract removal - sequential approach. Lens extraction should be performed in a second surgical procedure delayed for at least 6 months and ideally for 2 years, but with the penalty of significantly decreasing trabeculectomy filtering efficacy [47]. It should be kept in mind that in special cases - owing to increasing age, comorbidities or operative/anaesthetic contraindications - patients who are not candidates for two separate visits to an operation theatre can be considered for combined surgery. In this line of thought, there are those who support that, given the overall effect that phacoemulsification has on a previous filtration bleb, the final outcome of both surgical approaches (combined vs. sequential) will be very similar. The only clinical trial comparing both surgical approaches shows a similar IOP value. However, pre-operative IOP values are not mentioned and it is, therefore, impossible to come to any conclusion about the extent of IOP reduction seen with both procedures [48].

\section{Novel Surgical Approaches}

A range of novel surgical alternatives has emerged in recent years. These new techniques are minimally invasive, mostly intended to be combined with cataract surgery so as to provide an additional IOP reduction and a further decrease in the burden of glaucoma medication. These include endoscopic cyclophotocoagulation, trabecular micro-bypass stent (iStent), trabeculotomy ab interno (Trabectome), canaloplasty and two more recent ab interno procedures, the Hydrus and CyPass devices.

Endoscopic cyclophotocoagulation consists of laser coagulation of ciliary processes and, when combined with phacoemulsification, allows for an IOP reduction ranging from 17.6 to $46.9 \%$ [49]. However, the majority of data comes from retrospective, uncontrolled case reports, with only one randomized controlled study reporting a significant IOP and glaucoma medication decrease in the treatment group (17.6 vs. $4.2 \%$ decrease in IOP and 57.5 vs. $0 \%$ decrease in medication) during a 3-year follow-up [50]. Also, there is concern about the high rate of postoperative complications comparing to drainage devices.

The iStent (Glaukos Corp., Laguna Hills, Calif., USA) is a device that is internally inserted into Schlemm's canal, allowing the aqueous humour to bypass the trabecular meshwork from the anterior chamber directly into Schlemm's canal. The largest controlled, randomized clinical trial reported a high proportion of patients achieving an IOP $<21 \mathrm{~mm} \mathrm{Hg}$ (61 vs. $50 \%, \mathrm{p}=0.036$ ) at 2 years' follow- up [51]. Also, a decrease in IOP-lowering medication was detected in $81.3 \%$.

The ab interno trabeculotomy, or Trabectome (NeoMedix Inc., Tustin, Calif., USA), uses an electrocautery to remove a section of trabecular meshwork. Several retrospective, uncontrolled trials have reported an IOP decrease ranging from 4 to $7 \mathrm{~mm} \mathrm{Hg}$ with the combination of the Trabectome with phacoemulsification, as well as an important drop in the number of glaucoma medications [49]. However, evidence concerning the IOP-lowering effect of the Trabectome alone remains inconclusive.

Finally, canaloplasty (iScience Interventional Corp., Menlo Park, Calif., USA) consists of the cannulation of Schlemm's canal with consequent stretch of the trabecular meshwork and seems to be a novel approach with a higher IOP reduction ( $40 \%$ or $10 \mathrm{~mm} \mathrm{Hg}$ ) when combined with phacoemulsification; however, associated drawbacks are the development of conjunctival scarring that could limit the efficacy of a subsequent trabeculectomy and the higher rate of surgical complications [52].

Hydrus (Ivantis Inc., Irvine, Calif., USA) is a new Schlemm's canal scaffold that increases aqueous humour outflow. Preliminary results from a 6-month follow-up clinical trial show promising results (average $4.7 \mathrm{~mm} \mathrm{Hg}$ IOP reduction) due to its double mechanism of action perforation of the trabecular meshwork and dilation of Schlemm's canal [53]. The CyPass (Transcend Medical, Menlo Park, Calif., USA) is a polyamide implant inserted into the suprachoroidal space through the angle region to promote the uveoscleral outflow. Outcomes reported show an IOP reduction of $37 \%$ in medically uncontrolled eyes and a $50 \%$ decrease in glaucoma medications during a 6-month follow-up, with very few side effects [54].

These new methods are most suitable combined with phacoemulsification in mild to moderate primary openangle glaucoma (POAG) when lower magnitude IOP reductions are sufficient; however, solid randomized controlled clinical trials comparing these combined approaches to phacoemulsification alone are still lacking for the majority of these new techniques and, to date, it is impossible to discern their true potential for disease management.

\section{Conclusion}

There is evidence that cataract surgery results in a modest long-lasting decrease in IOP in both POAG and CACG patients. Due to the inherent anatomical charac- 
teristics of each type of glaucoma, this hypotensive effect is more prominent in eyes with CACG, in which lens extraction widens the anterior chamber. Phacoemulsification is becoming progressively more important for CACG control due to its ability to remodel anterior chamber anatomy and there is a current debate on whether clear lens extraction has a good risk-to-benefit ratio for CACG prevention and/or treatment.

On the other hand, for POAG-related ocular hypertension, primarily caused by trabecular meshwork dysfunction, cataract surgery results in a more limited IOP decrement, and its mechanism remains poorly understood. In these patients, this procedure has a minor impact on IOP control, but this modest and variable IOP decrease can still be clinically significant and help patients reach their target IOP. However, for the management of advanced glaucoma with uncontrolled IOP, priority should be given to a more aggressive IOP lowering strategy - such as filtering surgery - and cataract surgery should be delayed. Combined surgery - phacotrabeculectomy - is a surgical procedure without a formal indication, but might be useful for some situations. Future studies with well-designed, large, prospective randomized trials are still lacking, but current data suggests this widespread surgery will find its proper place in the practice pattern of glaucoma management.

\section{Disclosure Statement}

None of the authors has a conflict of interest with the submission and no financial support was received.

\section{References}

1 Pascolini D, Mariotti SP: Global estimates of visual impairment: 2010. Br J Ophthalmol 2012;96:614-618.

2 Quigley HA, Broman AT: The number of people with glaucoma worldwide in 2010 and 2020. Br J Ophthalmol 2006;90:262-267.

3 Asbell PA, Dualan I, Mindel J, Brocks D, Ahmad M, Epstein S: Age-related cataract. Lancet 2005;365:599-609.

4 Shrivastava A, Singh K: The effect of cataract extraction on intraocular pressure. Curr Opin Ophthalmol 2010;21:118-122.

5 Mansberger SL, Gordon MO, Jampel HD, Bhorade A, Brandt JD, Wilson B, Kass MA: Reduction in intraocular pressure after cataract extraction: the Ocular Hypertension Treatment Study. Ophthalmology 2012;119: 1826-1831.

6 Pohjalainen T, Vesti E, Uusitalo RJ, Laatikainen L: Intraocular pressure after phacoemulsification and intraocular lens implantation in nonglaucomatous eyes with and without exfoliation. J Cataract Refract Surg 2001; 27:426-431.

7 Shingleton BJ, Pasternack JJ, Hung JW, O'Donoghue MW: Three and five year changes in intraocular pressures after clear corneal phacoemulsification in open angle glaucoma patients, glaucoma suspects, and normal patients. J Glaucoma 2006;15:494498.

8 Jacobi PC, Dietlein TS, Lüke C, Engels B, Krieglstein GK: Primary phacoemulsification and intraocular lens implantation for acute angle-closure glaucoma. Ophthalmology 2002; 109:1597-1603.

9 Su W-W, Chen PY-F, Hsiao C-H, Chen HS-L: Primary phacoemulsification and intraocular lens implantation for acute primary angleclosure. PLoS One 2011;6:e20056.
10 Hayashi K, Hayashi H, Nakao F, Hayashi F: Effect of cataract surgery on intraocular pressure control in glaucoma patients. J Cataract Refract Surg 2001;27:1779-1786.

11 Lai JS, Tham CC, Chan JC, Lam DS: Phacotrabeculectomy in treatment of primary angleclosure glaucoma and primary open-angle glaucoma. Jpn J Ophthalmol 2004;48:408411.

12 Liu CJ, Cheng CY, Wu C-W, Lau L-I, Chou JC, Hsu W-M: Factors predicting intraocular pressure control after phacoemulsification in angle-closure glaucoma. Arch Ophthalmol 2006;124:1390-1394.

13 Lai JS, Tham CC, Chan JC: The clinical outcomes of cataract extraction by phacoemulsification in eyes with primary angle-closure glaucoma (PACG) and co-existing cataract: a prospective case series. J Glaucoma 2006; 15 : 47-52.

14 Kim DD, Doyle JW, Smith MF: Intraocular pressure reduction following phacoemulsification cataract extraction with posterior chamber lens implantation in glaucoma patients. Ophthalmic Surg Lasers 1999;30:3740.

15 Shingleton B, Gamell L, O’Donoghue MW, Baylus SL, King R: Long-term changes in intraocular pressure after clear corneal phacoemulsification: normal patients versus glaucoma suspect and glaucoma patients. J Cataract Refract Surg 1999;25:885-890.

16 Pohjalainen T, Vesti E, Uusitalo RJ, Laatikainen L: Phacoemulsification and intraocular lens implantation in eyes with open-angle glaucoma. Acta Ophthalmol Scand 2001;79: 313-316.

17 Mathalone N, Hyams M, Neiman S, Buckman G, Hod Y, Geyer O: Long-term intraocular pressure control after clear corneal phaco- emulsification in glaucoma patients. J Cataract Refract Surg 2005;31:479-483.

18 Poley BJ, Lindstrom RL, Samuelson TW, Schulze R: Intraocular pressure reduction after phacoemulsification with intraocular lens implantation in glaucomatous and nonglaucomatous eyes: evaluation of a causal relationship between the natural lens and openangle glaucoma. J Cataract Refract Surg 2009; 35:1946-1955.

19 Yang HS, Lee J, Choi S: Ocular biometric parameters associated with intraocular pressure reduction after cataract surgery in normal eyes. Am J Ophthalmol 2013;156:89-94.

20 Huang G, Gonzalez E, Lee R, Chen Y-C, He M, Lin SC: Association of biometric factors with anterior chamber angle widening and intraocular pressure reduction after uneventful phacoemulsification for cataract. J Cataract Refract Surg 2012;38:108-116.

21 Hayashi K, Hayashi H, Nakao F, Hayashi F: Changes in anterior chamber angle width and depth after intraocular lens implantation in eyes with glaucoma. Ophthalmology 2000; 107:698-703.

22 Issa S, Pacheco J, Mahmood U, Nolan J, Beatty S: A novel index for predicting intraocular pressure reduction following cataract surgery. Br J Ophthalmol 2005;89:543-546.

23 Liu CJ, Cheng C-Y, Ko Y-C, Lau L-I: Determinants of long-term intraocular pressure after phacoemulsification in primary angle-closure glaucoma. J Glaucoma 2011;20:566-570.

24 Pradhan S, Leffler CT, Wilkes M, Mahmood MA: Preoperative iris configuration and intraocular pressure after cataract surgery. J Cataract Refract Surg 2012;38:117-123.

25 Shrivastava A, Singh K: The impact of cataract surgery on glaucoma care. Curr Opin Ophthalmol 2014;25:19-25.
Review of the Intraocular PressureLowering Effects of Cataract Surgery
Ophthalmic Res 2015;53:141-148 DOI: $10.1159 / 000377635$ 
26 Wang N, Chintala SK, Fini ME, Schuman JS: Ultrasound activates the TM ELAM-1/IL-1/ NF- $\kappa B$ response: a potential mechanism for intraocular pressure reduction after phacoemulsification. Invest Ophthalmol Vis Sci 2003;44:1977-1981.

27 Poley BJ, Lindstrom RL, Samuelson TW: Long-term effects of phacoemulsification with intraocular lens implantation in normotensive and ocular hypertensive eyes. J Cataract Refract Surg 2008;34:735-742.

28 Guan H, Mick A, Porco T, Dolan BJ: Preoperative factors associated with IOP reduction after cataract surgery. Optom Vis Sci 2013;90: 179-184.

29 Foster PJ, Aung T, Nolan WP, Machin D, Baasanhu J, Khaw PT, Alsbirk P-H, Lee PS, Seah SKL, Johnson GJ: Defining 'occludable' angles in population surveys: drainage angle width, peripheral anterior synechiae, and glaucomatous optic neuropathy in east Asian people. Br J Ophthalmol 2004;88:486-490.

30 Lam DS, Leung DY, Tham CC, Li FC, Kwong YY, Chiu TY, Fan DS: Randomized trial of early phacoemulsification versus peripheral iridotomy to prevent intraocular pressure rise after acute primary angle closure. Ophthalmology 2008;115:1134-1140.

31 Husain R, Gazzard G, Aung T, Chen Y, Padmanabhan V, Oen FTS, Seah SKL, Hoh S-T: Initial management of acute primary angle closure: a randomized trial comparing phacoemulsification with laser peripheral iridotomy. Ophthalmology 2012;119:2274-2281.

32 Lachkar Y: Acute angle closure and angle closure glaucoma: phacoemulsification as firstline treatment (in French). J Fr Ophtalmol 2010;33:273-278.

33 Teekhasaenee C, Ritch R: Combined phacoemulsification and goniosynechialysis for uncontrolled chronic angle-closure glaucoma after acute angle-closure glaucoma. Ophthalmology 1999;106:669-674.

34 Tham CC, Kwong YY, Baig N, Leung DY, Li FC, Lam DS: Phacoemulsification versus trabeculectomy in medically uncontrolled chronic angle-closure glaucoma without cataract. Ophthalmology 2013;120:62-67.
35 AGIS Investigators: The Advanced Glaucoma Intervention Study: 8. Risk of cataract formation after trabeculectomy. Arch Ophthalmol 2001;119:1771-1779.

36 Friedman DS, Vedula SS: Lens extraction for chronic angle-closure glaucoma. Cochrane Database Syst Rev 2006;3:CD005555.

37 Thomas R, Walland MJ, Parikh RS: Clear lens extraction in angle closure glaucoma. Curr Opin Ophthalmol 2011;22:110-114.

38 Azuara-Blanco A, Burr JM, Cochran C, Ramsay C, Vale L, Foster P, Friedman D, Quayyum Z, Lai JS, Nolan W, Aung T, Chew P, McPherson G, McDonald A, Norrie J; Effectiveness in Angle-closure Glaucoma of Lens Extraction (EAGLE) Study Group: The effectiveness of early lens extraction with intraocular lens implantation for the treatment of primary angleclosure glaucoma (EAGLE): study protocol for a randomized controlled trial. Trials 2011; 12:133.

39 Tham CC, Kwong YY, Leung DY, Lam SW, Li FC, Chiu TY, Chan JC, Chan CH, Poon AS, Yick DW, Chi CC, Lam DS, Lai JS: Phacoemulsification versus combined phacotrabeculectomy in medically controlled chronic angle closure glaucoma with cataract. Ophthalmology 2008;115:2167-2173.

40 Tham CC, Kwong YY, Leung DY, Lam SW, Li FC, Chiu TY, Chan JC, Lam DS, Lai JS: Phacoemulsification versus combined phacotrabeculectomy in medically uncontrolled chronic angle closure glaucoma with cataracts. Ophthalmology 2009;116:725-731.

41 American Academy of Ophthalmology Glaucoma Panel: Preferred Practice Pattern ${ }^{\circledR}$ Guidelines. Primary Angle Closure. San Francisco, 2010.

42 Tsai H-Y, Liu CJ, Cheng C-Y: Combined trabeculectomy and cataract extraction versus trabeculectomy alone in primary angle-closure glaucoma. Br J Ophthalmol 2009;93: 943-948.

43 Cullen KA, Hall MJ, Golosinskiy A: Ambulatory Surgery in the United States, 2006. Hyattsville, 2009.
44 Lochhead J, Casson RJ, Salmon JF: Long term effect on intraocular pressure of phacotrabeculectomy compared to trabeculectomy. Br J Ophthalmol 2003;87:850-852.

45 Ogata-Iwao M, Inatani M, Takihara $\mathrm{Y}$, Inoue $\mathrm{T}$, Iwao K, Tanihara H: A prospective comparison between trabeculectomy with mitomycin $\mathrm{C}$ and phacotrabeculectomy with mitomycin C. Acta Ophthalmol 2013;91:e500-e501.

46 European Glaucoma Society: Terminology and Guidelines for Glaucoma, ed 3. Savona, Dogma, 2008.

47 Husain R, Liang S, Foster PJ, Gazzard G, Bunce C, Chew PTK, Oen FTS, Khaw PT, Seah SKL, Aung T: Cataract surgery after trabeculectomy: the effect on trabeculectomy function. Arch Ophthalmol 2012;130:165-170.

48 Donoso R, Rodríguez A: Combined versus sequential phacotrabeculectomy with intraoperative 5-fluorouracil. J Cataract Refract Surg 2000;26:71-74.

49 Budenz DL, Gedde SJ: New options for combined cataract and glaucoma surgery. Curr Opin Ophthalmol 2014;25:141-147.

50 Berke SJ: Endolaser cyclophotocoagulation in glaucoma management. Tech Ophthalmol 2006;4:74-81.

51 Craven ER, Katz LJ, Wells JM, Giamporcaro JE: Cataract surgery with trabecular microbypass stent implantation in patients with mild-to-moderate open-angle glaucoma and cataract: two-year follow-up. J Cataract Refract Surg 2012;38:1339-1345.

52 Augustinus CJ, Zeyen T: The effect of phacoemulsification and combined phaco/glaucoma procedures on the intraocular pressure in open-angle glaucoma. A review of the literature. Bull Soc Belge Ophtalmol 2012;51-66.

53 Radcliffe NM, Lynch MG, Brown RH: Ab interno stenting procedures. J Cataract Refract Surg 2014;40:1273-1280.

54 Hoeh H, Ahmed IIK, Grisanti S, Grisanti S, Grabner G, Nguyen QH, Rau M, Yoo S, Ianchulev T: Early postoperative safety and surgical outcomes after implantation of a suprachoroidal micro-stent for the treatment of open-angle glaucoma concomitant with cataract surgery. J Cataract Refract Surg 2013;39:431-437. 